\title{
Overexpression of microRNA-125b sensitizes human hepatocellular carcinoma cells to 5-fluorouracil through inhibition of glycolysis by targeting hexokinase II
}

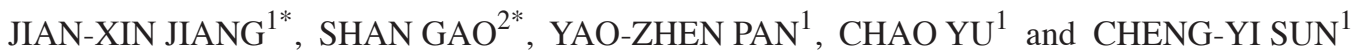 \\ Departments of ${ }^{1}$ Hepatobiliary Surgery and ${ }^{2}$ Digestive Internal Medicine, \\ Affiliated Hospital of Guiyang Medical College, Guiyang, Guizhou 550004, P.R. China
}

Received December 6, 2013; Accepted May 2, 2014

DOI: $10.3892 / \mathrm{mmr} .2014 .2271$

\begin{abstract}
FU)-based chemotherapy is widely used in the treatment of human hepatocellular carcinoma. However, despite impressive initial clinical responses, the majority of patients eventually develop resistance to 5-FU. The microRNA (miR)-125 family has been implicated in a variety of carcinomas as either a tumor suppressor or promoter. In the present study, the role of miR-125b in acquired 5-FU resistance in multiple human hepatocellular carcinoma cell lines was investigated using transfection of miR-125b. Compared with 5-FU-sensitive cells, 5-FU-resistant cells exhibited reduced expression levels of miR-125b. Furthermore, transfection of pre-miR-125b into liver cancer cells resulted in sensitization of 5-FU-resistant cells to 5-FU. In addition, the glucose uptake and lactate production in 5-FU-resistant liver cancer cells were demonstrated to be significantly increased compared with 5-FU-sensitive cells $(\mathrm{P}<0.05)$, indicating that targeting glycolytic pathways may overcome chemoresistance in human liver cancer cells. Notably, miR-125 was found to downregulate glucose metabolism by directly targeting hexokinase II. Since drug resistance is a common phenotype of malignant cancer cells, the finding that miR-125b expression levels are negatively correlated with 5-FU resistance in hepatocellular carcinoma cells is consistent with the reported functions of miR-125b. In conclusion, the present study identified miR-125b as a tumor suppressor-like microRNA, which exhibits great potential as a diagnostic and prognostic biomarker in hepatocellular carcinoma.
\end{abstract}

Correspondence to: Dr Cheng-Yi Sun, Department of Hepatobiliary Surgery, Affiliated Hospital of Guiyang Medical College, 28 Guiyi Street, Guiyang, Guizhou 550004, P.R. China

E-mail: chengyisun@medmail.com.cn

*Contributed equally

Key words: microRNA-125b, 5-fluorouracil, human hepatocellular carcinoma cells, glycolysis, hexokinase II

\section{Introduction}

MicroRNAs (miRNAs) are a family of $\sim 22$ nucleotide length small noncoding RNAs that regulate gene expression by translational repression, acting on the 3'-untranslated region (UTR) of target genes (1-3). A number of studies have demonstrated that miRNAs function as either tumor suppressors by downregulating oncogenic targets, or tumor promoters through negatively regulating tumor-suppressive target mRNAs $(4,5)$. In either way, miRNAs may be used as biomarkers for the early diagnosis of cancer and other diseases (6). In addition, miRNAs and the respective target genes may be utilized in anticancer therapy in addition to pharmaceutical applications (7). Among miRNA families with important functions, the miR-125 family has been reported to be implicated in a variety of carcinomas as either tumor suppressor or promoter (8). miR-125b has been revealed to exert tumor-suppressor functions in several cancer types, including ovarian (9), bladder (10) and breast cancer (11) as well as hepatocellular carcinoma (12), melanoma (13) and osteosarcoma (14). However, in contrast to its tumor-suppressive properties, miR-125b may also act as an oncogene in several cancers; for example, overexpression of miR-125b has been investigated in several cancer types, including pancreatic cancer (15) and prostate cancer (16).

In 1956, Otto Warburg observed that the rate of glycolysis was abnormally high in cancer cells, yet a smaller fraction of this glucose was broken down by oxidative phosphorylation (17). This 'Warburg effect' indicates that cancer cells prefer glycolytic breakdown of glucose for energy, rather than mitochondrial oxidative phosphorylation, suggesting that targeting cancer cell metabolism may be an effective approach to selectively treating malignant cancer cells (18). Furthermore, dysregulated metabolism of cancer cells has been associated with drug resistance (19). Chemoresistant cells usually exhibit an upregulated glucose metabolism.

Lactate dehydrogenase A (LDHA), an enzyme involved in anaerobic glycolysis, has been reported to contribute to paclitaxel resistance in breast cancer (20), and glycolysis suppression through hexokinase II (HK II) inhibition was found to enhance the cytotoxic effects of daunorubicin and doxorubicin in vitro and in vivo (21). In addition, the pyruvate dehydrogenase kinase inhibitor dichloroacetic acid (DCA) potentiated the anticancer 
effects of 5-fluorouracil (5-FU) through initiating the mitochondrial apoptosis pathway (22), suggesting that targeting the glucose metabolism of cancer cells contributes to the sensitization of cancer cells to therapeutic agents.

Hepatocellular carcinoma (HCC) is an aggressive and highly vascular tumor (23). Currently, the treatment options for HCC depend on the stage and grade of the tumor. 5-FU-based chemotherapy is widely used in the treatment of multiple cancer types, and systemic therapy with 5-FU alone or in combination with other chemotherapeutic agents has been utilized in the treatment of $\operatorname{HCC}(24,25)$. However, despite impressive initial clinical responses, the majority of patients eventually develop resistance to 5-FU. The mechanisms of 5-FU resistance in HCC remain under investigation; 5-FU-resistant colon cancer cells have been reported to exhibit upregulated glucose metabolism, and the combination of DCA and 5-FU treatment exerts a synergistic inhibitory effect (22), indicating a close correlation between dysregulated glucose metabolism and 5-FU resistance.

In the present study, the tumor suppressor activities of miR-125b were investigated in human HCC cells.

\section{Materials and methods}

Cell lines and cell culture. The SMMC-7221, Huh7, MHCC-97L, HepG2, HepG3 and BEL-7402 human HCC cell lines, along with THLE-2 and THLE-3 normal human liver cell lines were obtained from the American Type Culture Collection (Manassas, VA, USA). Human HCC cells were grown in Williams' medium E (\#W1878; Sigma-Aldrich, Hong-Kong, China) supplemented with $10 \%$ fetal bovine serum (\#10099-141; Gibco-BRL, Carlsbad, CA, USA) and antibiotics. The THLE- 2 and THLE- 3 cells were cultured in LHC-8 medium (\#12678-017; Gibco-BRL) supplemented with $70 \mathrm{ng} / \mathrm{ml}$ phosphoethanolamine (\#P0503; Sigma-Aldrich), $5 \mathrm{ng} / \mathrm{ml}$ epidermal growth factor (\#E5036; Sigma-Aldrich), $10 \%$ fetal bovine serum and penicillin-streptomycin $(10,000$ $\mathrm{U} / \mathrm{ml}, 10 \mathrm{ml} /$ tube stored at $-20^{\circ} \mathrm{C}$; \#15140-122; Gibco-BRL). All cells were cultured in a humidified atmosphere of $5 \% \mathrm{CO}_{2}$ in air at $37^{\circ} \mathrm{C}$. 5 -FU-resistant clone 1 , clone 2 and pool cells were developed from HepG3 cells by treatment with gradually increasing concentrations of 5-FU (\#F6627; Sigma-Aldrich) in cell culture medium. The resistant cells were re-selected every month through doxorubicin treatment.

Antibodies and reagents. Anti-HK II (rabbit monoclonal, \#2867) and anti- $\beta$-actin (rabbit monoclonal, \#4967) antibodies were purchased from Cell Signaling Technology, Inc. (Danvers, MA, USA). Anti-5-FU antibodies were provided by Sigma-Aldrich.

Glucose uptake assay. Cells were seeded in 12-well plates at $1 \times 10^{5}$ to $3 \times 10^{5}$ cells per well. The culture media was collected at $48 \mathrm{~h}$ and stored at $-20^{\circ} \mathrm{C}$. Glucose uptake was measured using an Amplex Red Glucose/Glucose Oxidase assay kit (Molecular Probes, Camarillo, CA, USA). Absorbance was measured at $563 \mathrm{~nm}$ using a SpectraMax M5 plate reader (Molecular Devices, Sunnyvale, CA, USA) and the results were normalized to the quantity of total glucose compared with that of the control cells.
Lactate production assay. Lactate production in the medium was detected by using a Lactate assay kit (BioVision, Mountain View, CA, USA). The results were normalized to the quantity of total lactate compared with the control cells.

Pre-miRNA or anti-miRNA transfection. miRNA precursors (pre-miRNAs) and miRNA antisense RNAs (anti-miRNAs) were purchased from Applied Biosystems China (Beijing, China). Pre-miR-negative (\#AM17110; Ambion, Austin, TX, USA) served as negative controls. Lipofectamine 2000 (Invitrogen Life Technologies, Carlsbad, CA, USA) was used for the pre-miRNAs or anti-miRNA transfections. At $48 \mathrm{~h}$ after transfection, the expression levels of miR-125b were detected by quantitative polymerase chain reaction and the expression levels of HK II, a target of miR-125b, were analyzed by western blot analysis.

Plasmid DNA transfection. Transfection was performed using Lipofectamine 2000 transfection reagent (Invitrogen Life Technologies) according to the manufacturer's instructions. Overexpression vector containing wild-type HK II (Myc-DDK-tagged; RC209482) was purchased from Origene Technologies, Inc. (Rockville, MD, USA). At $48 \mathrm{~h}$ following transfection, the cells were collected for further analysis, and an aliquot was used to prepare whole-cell lysates.

Generation of the HepG3 5-FU-resistant cell line. HepG3 cells were treated with gradually increasing concentrations of 5-FU under regular cell culture conditions for the selection of resistant cells. Following successive treatments for up to two months, several resistant cell clones were developed from the parental cell line. 5-FU-resistant clone 1, clone 2 and pooled clones were used for subsequent experiments. The resistant cells were selected by 5 -FU treatments each month.

Reverse transcription (RT)-PCR. RNA was isolated from cultured cells using an RNeasy mini-kit (Qiagen, Hilden, Germany) with an on-column DNAse digestion step according to the manufacturer's instructions. Briefly, the lysates of the cells were passed through a Qiashredder (Qiagen) and the eluted lysates were mixed 1:1 with $70 \%$ ethanol. The lysates were applied to a mini-column and subsequent to washing and DNAse I digestion, the RNAs were eluted in 30-50 $\mu \mathrm{l}$ RNAse-free water. The quantity and quality of total RNA samples was checked by agarose-gel-electrophoresis and a Bioanalyzer RNA 6000 Nano assay (Agilent, Waldbronn, Germany). For miRNA expression analysis, RT-PCR was performed using a TaqMan microRNA reverse transcription kit (Applied Biosystems, China) and TaqMan microRNA assays kit (Applied Biosystems) following the manufacturer's instructions. The precursor miR-125b RT primer used was: GTCGTATCCAGTGCAGGGTCCGAG GTATTCGCACTGGATACGACAGCACG. RNU6B (RT primer, TCGTATCCAGTGCAGGGTCCGAGGTATTCG CACTGGATACGACAAAATATGGAAC; Invitrogen Life Technologies) served as an internal control. All reactions were performed in triplicate. End-point PCR was conducted to analyze the expression levels of miR-125b using a mirVana RT-PCR miRNA detection kit and mirVana RT-PCR Primer sets (\#AM1558; Applied Biosystems China) according to 
A

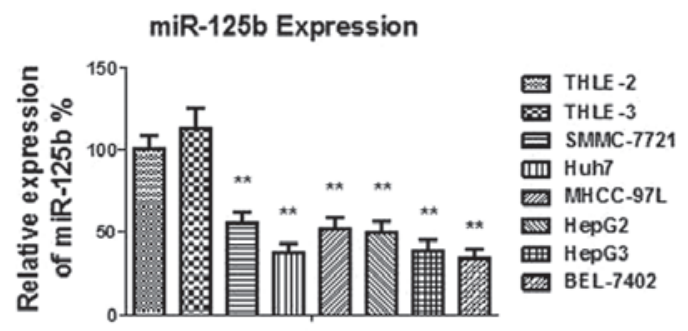

C

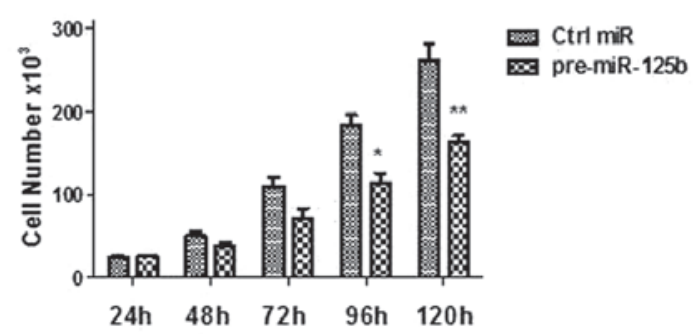

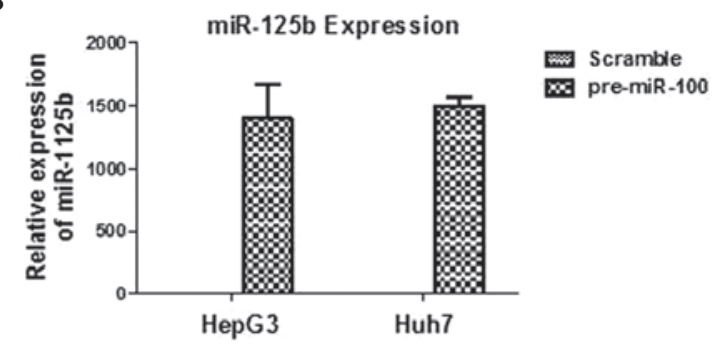

Huh7

Figure 1. miR-125b acts as a tumor suppressor in human hepatocellular carcinoma (HCC) cells. (A) Expression levels of miR-125b in multiple HCC cell lines compared with the normal human liver cell lines THLE-2 and THLE-3. (B) Expression levels of miR-125b after transfection with pre-miR-125b in HepG3 and Huh7 HCC cells. (C) Overexpression of miR-125b suppressed HCC cell growth. Columns, mean of three independent experiments; bars, standard error of the mean. ${ }^{*} \mathrm{P}<0.05 ;{ }^{* *} \mathrm{P}<0.01$ vs. control. miR, microRNA; Ctrl, control.

the manufacturer's instructions. Human U6 (Invitrogen Life Technologies)served as an internal control. All reactions were performed in triplicate. The relative quantities of mRNA were calculated using the comparative CT method.

Luciferase reporter assay. The pMIR-reporter luciferase vector containing either the wild-type HK II 3'-UTR or the binding site mutant HK II 3'-UTR and the empty vector were constructed according to methods previously described (29). For the luciferase assay, cells were seeded at a density of $2 \times 10^{5}$ per well in 24 -well plates and co-transfected with pMIR-REPORT luciferase reporters (\#AM5795; Ambion) with the 3'-UTRs of wild-type HK II or binding site mutant HK II, and pre-miR-125b or pre-miR-negative using Lipofectamine 2000 reagent. After $48 \mathrm{~h}$, the cells were harvested and lysed with passive lysis buffer (Promega Corporation, Madison, WI, USA). Luciferase activity was measured using a dual luciferase reporter assay (Promega Corporation). The pRL-TK vector (Promega Corporation) served as an internal control. The results were expressed as relative luciferase activity (firefly luciferase/renilla luciferase).

Cell viability assay. The cancer cells were treated with 5-FU at the indicated concentrations for $48 \mathrm{~h}$. The cells were seeded in a 48 -well plate, at a density of $1 \times 10^{4}$ cells/well in $0.2 \mathrm{ml}$ Williams' medium E or LHC-8 medium containing $10 \%$ fetal bovine serum. Following overnight incubation under the same cultivating conditions, each well was refreshed with serum-free medium (SFM) for another day. The cells were then treated with SFM containing various concentrations of 5-FU. The drug-containing SFM was refreshed after two days and incubated under the same conditions. Cell viability was accessed by MTT (Sigma Diagnostics, Inc., St. Louis, MO, USA) staining for $1 \mathrm{~h}$ followed by the addition of DMSO and by measuring the absorbance at $590 \mathrm{~nm}$ with a SpectraMax Plus384 plate reader (Molecular Devices). The relative viability was obtained from the absorbance at $590 \mathrm{~nm}$ of drug-treated cells/the absorbance at $590 \mathrm{~nm}$ of untreated cells. The same experiment was repeated three times.

Western blot analysis. The cells were harvested and lysed in a buffer containing $50 \mathrm{mM}$ Tris- $\mathrm{HCl}, \mathrm{pH} 7.5,150 \mathrm{mM} \mathrm{NaCl}$, $2 \mathrm{mM}$ EDTA, 1\% Triton, $1 \mathrm{mM}$ phenylmethanesulfonyl fluoride and protease inhibitor cocktail (Sigma Diagnostics, Inc.) for $20 \mathrm{~min}$ on ice. The lysates were cleared by centrifugation at $10,000 \mathrm{x} \mathrm{g}$ at $4^{\circ} \mathrm{C}$ for $10 \mathrm{~min}$. The supernatants were collected and protein concentrations were determined by the Bradford assay (Bio-Rad, Hercules, CA, USA). The proteins were then separated by SDS-PAGE and transferred to a nitrocellulose membrane (Bio-Rad). Subsequent to blocking with $5 \%$ non-fat dry milk in phosphate-buffered saline (PBS) for $1 \mathrm{~h}$, the membranes were incubated overnight at $4-8^{\circ} \mathrm{C}$ with the primary antibodies in PBS with $5 \%$ non-fat dry milk. The membranes were extensively washed with PBS and incubated with horseradish peroxidase-conjugated secondary anti-mouse antibody or anti-rabbit antibody (1:2,000; Bio-Rad). Following additional washes with PBS, the antigen-antibody complexes were visualized with an enhanced chemiluminescence kit (Pierce Biotechnology, Inc., Rockford, IL, USA). The LAS-3000 Imaging system (Fuji, Tokyo, Japan) was used for the membrane exposure.

Statistical analysis. Statistical evaluation for data analysis was determined by unpaired Student's t-test and GraphPad Prism software, version 5.0 (GraphPad Software, Inc., La Jolla, CA, 
$\mathbf{A}$ HepG3

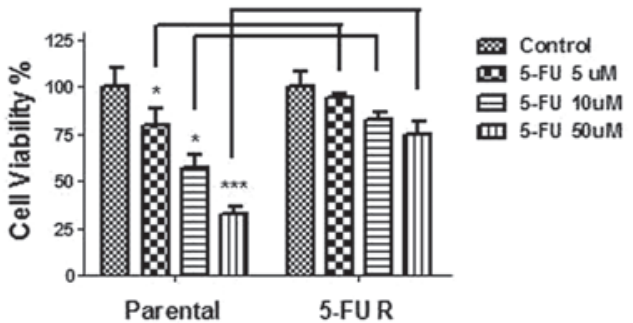

B

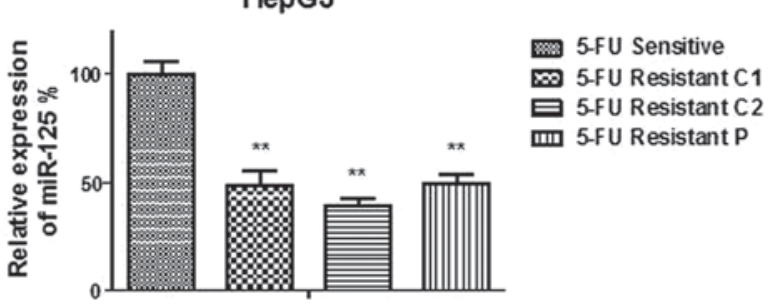

Figure 2. miR-125b is downregulated in 5-FU-resistant hepatocellular carcinoma cells. (A) Generation of 5-FU-resistant cells from HepG3. Parental cells were treated with gradually increasing concentrations of 5-FU in regular cell culture conditions for selection of resistant cells. HepG3 5-FU-resistant clones were pooled and treated with 5-FU at the indicated concentrations for $48 \mathrm{~h}$. (B) Expression levels of miR-125b in HepG3 5-FU-sensitive cells and resistant clone1, clone2 and pool cells. Columns, mean of three independent experiments; bars, standard error of the mean. ${ }^{*} \mathrm{P}<0.05$; ${ }^{* * * *} \mathrm{P}<0.001$, vs. 5-FU-resistant group. 5-FU, 5-fluorouracil; miR, microRNA; R, resistant pool group ; $\mathrm{C} 1 / 2$, clone $1 / 2$; $\mathrm{P}$, resistant pool group.

A

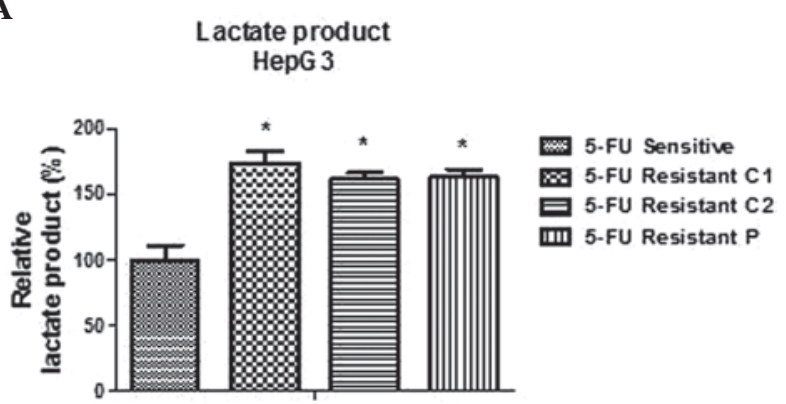

B

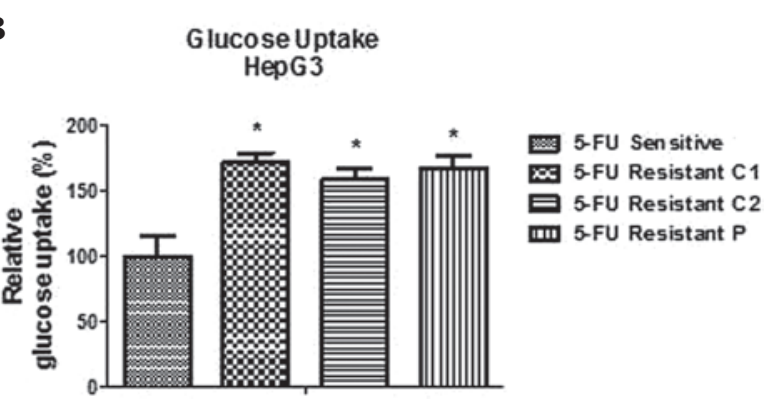

Figure 3. 5-fluorouracil (5-FU)-resistant cells exhibit upregulated glucose metabolism. (A) Lactate production and (B) glucose uptake were measured in HepG3 5-FU-sensitive and -resistant clone1, clone2 and pool cells. The relative glucose uptake and lactate production in 5-FU-resistant cells were normalized to the levels in 5-FU-sensitive cells. Columns, mean of three independent experiments; bars, standard error of the mean. " $\mathrm{P}<0.05$ vs. control. 5-FU, 5-fluorouracil; $\mathrm{Cl} / 2$, clone 1/2; $\mathrm{P}$, resistant pool group.

USA) was used. All data are shown as the means. $\mathrm{P}<0.05$ was considered to indicate a statistically significant difference.
A

hsa-miR-125b/HK2 Al ignment

3' agugUUCAAUCCCAGAGUCCCu 5' hsa-miR-125b II| II IIIIII|

1357:5' agCCAA-AUAAAACCUCAGGGa 3' HK2

B

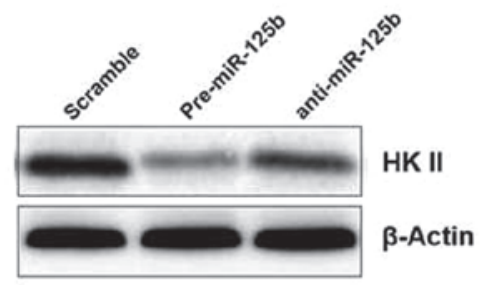

C HepG3

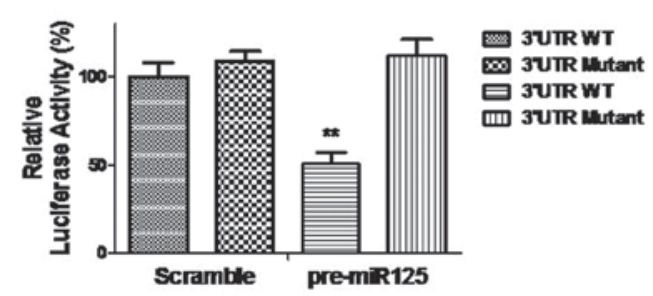

Figure 4. HK II is a direct target of miR-125b in hepatocellular carcinoma cells. (A) Target prediction with Targetscan.org: The 3'UTR of HK II contains putative binding sites for miR-125b. (B) HepG3 cells were transfected with $200 \mathrm{nM}$ pre-miR-negative (Scramble), pre-miR-125b or anti-miR-125b for $48 \mathrm{~h}$. Cell lysates were prepared for Western blot analysis. $\beta$-actin served as a loading control. (C) HepG3 cells were co-transfected with luciferase reporter plasmids with WT HK II 3'-UTR or mutant HK II 3'-UTR, and pre-miR-125b or pre-miR-negative (Scramble) using Lipofectamine 2000 reagent. At $48 \mathrm{~h}$ post-transfection, the cells were harvested and lysed with passive lysis buffer. Luciferase activities were measured by dual luciferase reporter assay. The pRL-TK vector served as an internal control. The results were expressed as relative luciferase activity (firefly luciferase/renilla luciferase). Columns, mean of three independent experiments; bars, standard error of the mean. ${ }^{* *} \mathrm{P}<0.01$ vs. control. HK, hexokinase; hsa, Homo sapiens; UTR, untranslated region; WT, wild-type; miR, microRNA.

\section{Results}

miR-125b acts as a tumor suppressor in human HCC cells. miR-125b has been reported to act as a tumor suppressor in multiple tumors. In the present study, the expression levels of miR-125b in multiple types of human HCC cells were compared with those in normal human hepatocyte cells. A significant reduction in the expression levels of miR-125b was observed in the HCC cell lines compared with the normal hepatocytes (Fig. 1A), suggesting that miR-125b may be a tumor suppressor in human liver cancer cells. The observation that miR-125b was downregulated in liver cancer cells prompted investigation into the detailed functions of miR-125b through transient transfection of miR-125b into two liver cancer cell lines, HepG3 and Huh7 (Fig. 1B). The cell growth rates were significantly reduced in miR-125b-overexpressing liver cancer cells (Fig. 1C), suggesting that miR-125b acts as a tumor suppressor in human HCC cells.

miR-125b is downregulated in 5-FU-resistant HCC cells. As discussed above, chemotherapy resistance is a major obstacle that limits the effectiveness of clinical management of liver cancer. The dysregulated expression of tumor suppressor genes 
A

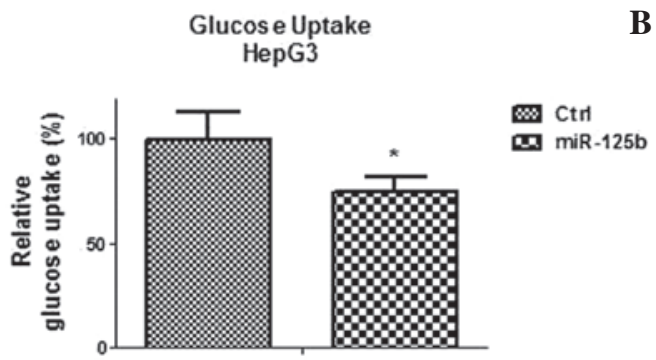

B

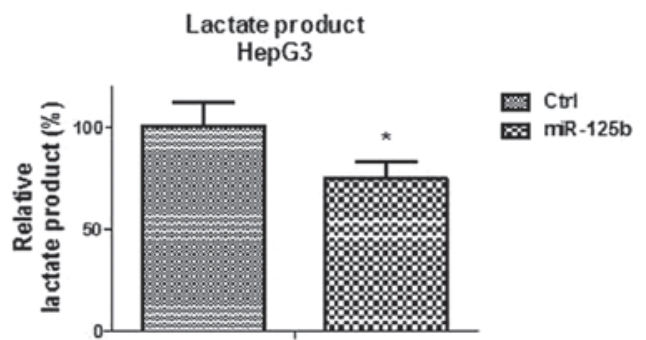

Figure 5. Overexpression of miR-125b in HepG3 cells reduces glucose metabolism. (A) Glucose uptake and (B) lactate production were measured in miR-125b-overexpressing HepG3 cells compared with the Ctrl group. The relative glucose uptake and lactate production of miR-125 overexpressing cells were normalized to the levels in negative control cells. Columns, mean of three independent experiments; bars, standard error of the mean. ${ }^{*} \mathrm{P}<0.05 \mathrm{vs.}$ control. Ctrl, control; miR, microRNA.

A

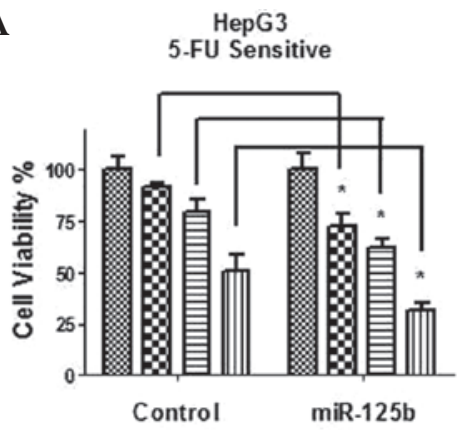

B

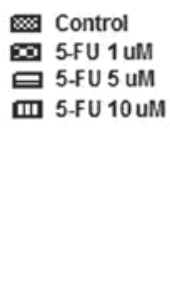

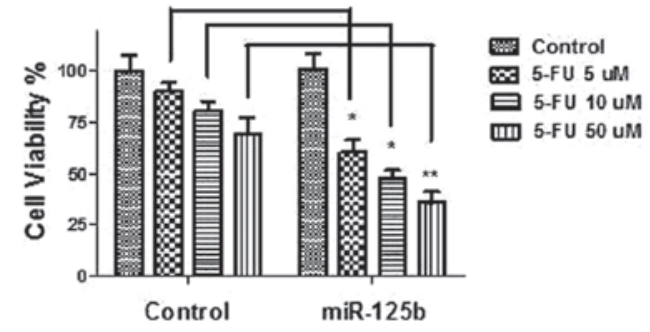

Figure 6. Overexpression of miR-125b in hepatocellular carcinoma cells sensitizes 5-FU-sensitive and 5-FU-resistant cells to 5-FU. (A) Overexpression of miR-125b inhibited HepG3 cell viability. 5-FU-sensitive cells were transfected with pre-miR-125b for $48 \mathrm{~h}$, then seeded into 48 -well plates and treated with the indicated concentrations of 5-FU followed by measurement of cell viability. (B) HepG3 5-FU-resistant cells were transfected with negative control and pre-miR-125b for $48 \mathrm{~h}$. The cells were seeded into 48 -well plates for 5-FU treatments at the indicated concentrations for $48 \mathrm{~h}$ followed by cell viability assays Columns, mean of three independent experiments; bars, standard error of the mean. ${ }^{*} \mathrm{P}<0.05$; ${ }^{* * *} \mathrm{P}<0.01$, vs. control. 5-FU, 5-fluorouracil; miR, microRNA.

negatively correlates with chemoresistance of cancer cells. To investigate the function of miR-125b in liver cancer cell chemosensitivity, a 5-FU-resistant cancer cell line was generated using HepG3 cells by gradually treating the cells with elevated concentrations of 5-FU. Two HepG3 5-FU-resistant colonies were selected and the remainder were combined as a 5-FU-resistant pool. Fig. 2A shows that the resistant cells were significantly less sensitive to regular 5 -FU treatments compared with the parental cells. The parental cells exhibited a $\mathrm{IC}_{50}$ at $\sim 10 \mu \mathrm{M}$, while the viability of 5-FU-resistant cells at $10 \mu \mathrm{M}$ was significantly higher $(\mathrm{P}<0.05$; Fig. $2 \mathrm{~A})$. The expression levels of miR-125b were measured in the 5-FU-resistant clone1, clone2 and pool cells. The results revealed that miR-125b was significantly downregulated in all 5-FU-resistant clones (Fig. 2B), suggesting that miR-125b functions as a tumor suppressor in liver cancer cells.

5-FU-resistant liver cancer cells exhibit increased glucose metabolism. In accordance with the Warburg effect, evidence supports the hypothesis that dysregulated cellular metabolism is associated with drug resistance in cancer therapy. To analyze the putative mechanisms for miR-125b-mediated sensitization to chemotherapy in HCC cells, the glucose metabolism in 5-FU-resistant and-sensitive cells was measured in the present study. Notably, glucose metabolism, which is normally upregulated in chemoresistant cancer cells, was markedly increased in 5-FU-resistant cells. Lactate production $(\mathrm{P}<0.05$; Fig. $3 \mathrm{~A})$ and glucose uptake $(\mathrm{P}<0.05$; Fig. $3 \mathrm{~B})$ were significantly increased in 5-FU-resistant cells, indicating that dysregulated metabolism may contribute to chemoresistance.

$H K$ II is a direct target of miR-125b. To further elucidate the mechanism for miR-125b-mediated sensitization to 5-FU in liver cancer cells, potential targets of miR-125b were investigated by searching miRNA databases. The three public miRNA databases (TargetScan, Pictar and MicroRNA) all predicted HK II to be a target of miR-125b; the 3'-UTR of HK II contains a highly conserved binding site for miR-125b (Fig. 4A). HKs catalyze the first committed step in glucose metabolism and are important in tumor initiation and maintenance. Thus far, to the best of our knowledge, no publication has reported HK II to be a direct target of miR-125b in HCC cells. To determine whether miR-125b affects HK II in chondrosarcoma cells, pre-miR-125b and anti-miR-125b were transfected into HepG3 cells. The overexpression of miR-125b markedly downregulated HK II protein expression levels in comparison with a control group, but inhibition of miR-125b restored HK II expression levels in HepG3 cells. (Fig. 4B). To identify whether miR-125b directly targets the 3'-UTR of HK II mRNA, luciferase reporter analysis was performed by co-transfecting the cells with a vector containing pMIR reporter-luciferase fused with the original sequence or a predicted binding site mutant of the 3'-UTR of HK II mRNA, and pre-miR-100 or control microRNA. Overexpression of miR-125b significantly reduced the luciferase activity of the reporter with wild-type HK II 3'-UTR by $\sim 60 \%$ in HepG3 
A

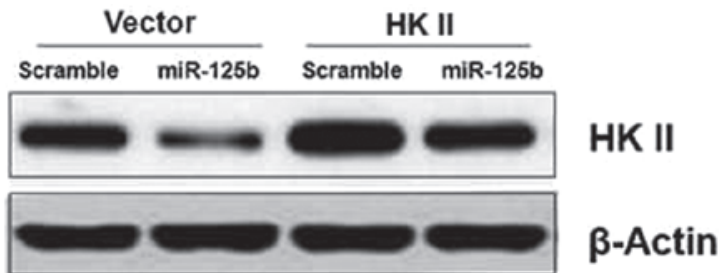

B

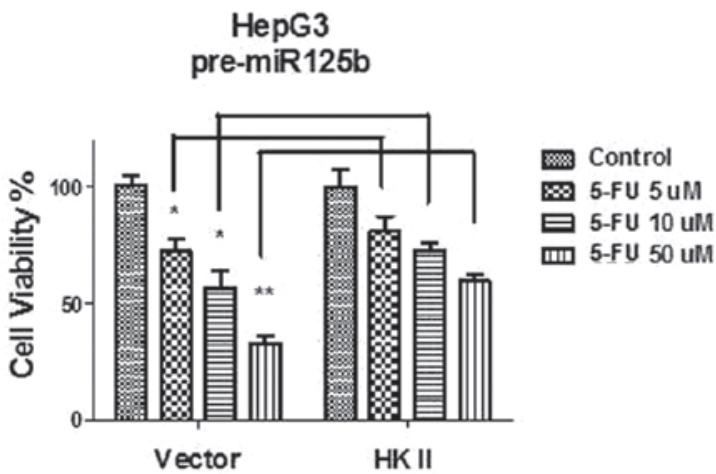

Figure 7. Restoration of HK II in miR-125b-overexpressing cells renders HepG3 cells resistant to 5-FU. (A) HepG3 cells were transfected with $200 \mathrm{nM}$ pre-miR-negative control and pre-miR-125b for $48 \mathrm{~h}$ followed by transfection with a vector control and an overexpression vector containing wild-type HK II for $24 \mathrm{~h}$. Cells were then collected and prepared for western blot analysis using anti-HK II antibody. $\beta$-actin served as a loading control. (B) HepG3 cells were transfected with pre-miR-125b and HK II as described above, then the cells were collected and seeded in 48-well plates for 5-FU treatment at the indicated concentrations for $48 \mathrm{~h}$. Cells were assessed by cell viability assays. Columns, mean of three independent experiments; bars, standard error of the mean. ${ }^{*} \mathrm{P}<0.05 ;{ }^{* *} \mathrm{P}<0.01$, vs. control. HK, hexokinase; miR, microRNA, 5-FU, 5-fluorouracil.

cells $(\mathrm{P}<0.01$; Fig. 4C). No inhibitory effects of miR-125b on the activity of the reporter with binding site mutant HK II 3'-UTR were detected (Fig. 4C). In conclusion, these results demonstrated that HK II is a direct target of miR-125b in HCC cells.

Overexpression of miR-125b in liver cancer cells inhibits glucose metabolism. The above results revealed that HK II is a direct target of miR-125b. HK II has been established as essential in catalyzing the first committed step in glucose metabolism. To identify whether miR-125b regulates glucose metabolism in liver cancer cells, glucose uptake and lactate production in miR-125b-overexpressing HepG3 cells were compared with those in cells transfected with control microRNA. The data revealed significant inhibitions of glucose uptake and lactate production in the miR-125b-overexpressing cells $(\mathrm{P}<0.05$; Fig. 5A and $\mathrm{B})$, indicating that miR-125b may sensitize liver cancer cells to 5-FU through downregulation of glucose metabolism. In conclusion, the results in Figs. 3 and 5 revealed a correlation between miR-125b-mediated suppression of glucose metabolism and 5-FU sensitivity.

Overexpression of miR-125b resensitizes 5-FU-resistant HCC cells through the inhibition of glucose metabolism. To determine the mechanisms accounting for miR-125b-mediated 5-FU sensitivity, miR-125b and control microRNA were transfected into HepG3 5-FU-sensitive and -resistant cells, and the cells were then treated with the indicated concentrations of 5-FU for
$48 \mathrm{~h}$. The effect of miR-125b overexpression on cell viability was examined following 5-FU administration. Treatment with 5-FU resulted in a marginal inhibition of cell viability in the control 5-FU-sensitive and -resistant HepG3 cells (Fig. 6A and B). However, when miR-125b was overexpressed in 5-FU-sensitive and -resistant liver cancer cells, the susceptibility of the cells to 5-FU was significantly increased (Fig. 6A and B), suggesting that miR-125 is involved in the development of 5-FU resistance. To examine whether sensitization to 5 -FU by miR-125b occurred through inhibition of the glucose metabolism, an overexpression vector containing wild-type HK II was transfected into miR-125 pre-transfected HepG3 cells. Exogenous overexpression of HK II in miR-125 pre-transfected cells restored the expression levels of HK II to non-transfection levels (Fig. 7A). The sensitivity of the transfected HepG3 cells to 5-FU was then measured (Fig. 7B). The results demonstrated that exogenous upregulation of the glucose metabolism by overexpression of HK II rendered miR-125b overexpressing HepG3 cells significantly more resistant to 5-FU in comparison to cells not transfected with HK II. In conclusion, the results suggested that the overexpression of miR-125b sensitized HCC cells to 5-FU through inhibition of HK II.

\section{Discussion}

The 'Warburg effect' describes the unique metabolic phenomenon where tumor cells preferentially utilize the markedly less efficient glycolytic process for energy production, even in the presence of oxygen $(17,18)$. A high rate of glycolysis in cancer has been observed, occurring through upregulation of the key enzymes HK II, glucose transporter 1, pyruvate kinase M2 isoform (PKM2), pyruvate dehydrogenase kinase 1 and LDHA (19). The ability to reduce the metabolic rate in cancer cells has been investigated as one mechanism of cancer suppressors. Evidence supports the hypothesis that dysregulated cellular metabolism is associated with drug resistance in cancer therapy (19). Researchers have attempted to use the unique bioenergetic properties of cancer cells to enhance the therapeutic efficacy of cytotoxic chemotherapy. In the glycolytic pathway, LDHA has been shown to contribute to paclitaxel/trastuzumab resistance in breast cancer (20), and inhibition of HK II was demonstrated to restore drug sensitivity in doxorubicin-resistant colon cancer cells (21). Therefore, targeting cancer cell metabolism with glycolysis inhibitors administered in combination with conventional chemotherapeutic agents may provide a synergistic inhibitory effect in the treatment of 5-FU-resistant liver cancer cells.

miRNAs have been identified as exhibiting oncogenic and tumor suppressor-like functions in the processes of cellular proliferation, differentiation and apoptosis. microRNAs, including miR-221 (26), miR-222 (27) and miR-106b (28), have previously been reported to function as oncogenes. Conversely, the majority of downregulated microRNAs, including miR-16 (29), miR-100 (30), miR-29a/b (31) and $\mathrm{miR}-146 \mathrm{a} / \mathrm{b}$ (32), have been reported to act as tumor suppressors. Increasing evidence has demonstrated that miR-125b is crucial in diverse cellular processes and numerous carcinomas. However, little is known regarding the role of miRNAs in chemoresistance to drugs such as 5-FU. In the present study, the differential expression profiles of miR-125b in normal 
human liver cells and human HCC cells were examined. miR-125b was found to be downregulated in liver cancer cells and overexpression of miR-125b significantly suppressed liver cancer cell growth, supporting the conclusion that miR-125b acts as a tumor suppressor in human HCC cells.

Thus far, several targets of miR-125b in cancers have been identified. The ErbB2 and ErbB3 oncogenes were reported to be targets of miR-125b in breast cancer cells (11). Other studies have shown that the tumor suppressor p53 is a direct target of miR-125b, and suppression of p53 gene expression by overexpression of miR-125b inhibits p53-dependent apoptosis in human neuroblastoma cells and human lung fibroblast cells (33). Furthermore, miR-125b has been reported to reduce the expression levels of the MUC1 oncoprotein, whose silencing promotes DNA damage-induced apoptosis in breast cancer cells (34).

HK II catalyzes the first irreversible step of the glycolytic pathway, where glucose is phosphorylated to glucose-6-phosphate with consumption of adenosine triphosphate. In addition to miR-125b, cancer glycolysis has also been reported to be regulated by miR-143, via the targeting of HK II (35). Furthermore, PKM2 is a target of the tumor-suppressive microRNA-326 and regulates the survival of glioma cells (36), demonstrating that targeting metabolic enzymes by microRNA results in downregulation of cancer cell metabolic processes. 5-FU is a widely used chemotherapeutic agent for the treatment of several types of cancer. 5-FU resistance may result in subsequent recurrence and metastasis of cancer, ultimately causing cancer-associated mortality. Although extensive investigations have been conducted with regard to 5-FU resistance in cancer cells, the specific mechanisms involved remain poorly understood. In the present study, HK II was identified as a direct target of miR-125b in HCC cells. Inhibition of HK II by overexpression of miR-125b resulted in suppression of the glucose metabolism pathway, which sensitized liver cancer cells to 5-FU. Restoration of HK II by exogenous transfection of HK II rendered liver cancer cells resistant to 5-FU, indicating that overexpression of miR-125b sensitizes 5-FU-resistant HCC cells through the inhibition of HK II.

In conclusion, the role of miR-125b in acquired 5-FU resistance was investigated in multiple human HCC cell lines. Compared with 5-FU-sensitive cells, 5-FU-resistant cells exhibited reduced expression levels of miR-125b, and transfection of pre-miR-125b into liver cancer cells resulted in an increased sensitivity of 5-FU-resistant cells to 5-FU. Since drug resistance is a phenotype of malignant cancer cells, the finding that miR-125b expression levels are negatively correlated with 5-FU resistance in HCC cells is consistent with the reported functions of miR-125b. In addition, 5-FU-resistant cells exhibited higher glucose metabolic activity than 5-FU-sensitive cells, and miR-125 was identified to downregulate glucose metabolism by directly targeting HK II. These results identified miR-125b as a tumor suppressor-like microRNA, which has great potential as a diagnostic and prognostic biomarker.

\section{Acknowledgements}

The authors would like to thank Dr Chao Yu at the Department of Hepatobiliary Surgery, Affiliated Hospital of Guiyang
Medical College for editorial assistance. This study received support from the Chinese National Natural Science Foundation (grant no. 81160311) and the Chinese Postdoctoral Foundation (grant no. 2013-52-134523).

\section{References}

1. Ameres SL and Zamore PD: Diversifying microRNA sequence and function. Nat Rev Mol Cell Biol 14: 475-488, 2013.

2. Bushati N and Cohen SM: microRNA functions. Annu Rev Cell Dev Biol 23: 175-205, 2007.

3. Di Leva G, Garofalo M and Croce CM: MicroRNAs in Cancer. Annu Rev Pathol 9: 287-314, 2014.

4. Shenouda SK and Alahari SK: MicroRNA function in cancer: oncogene or a tumor suppressor? Cancer Metastasis Rev 28: 369-378, 2009.

5. Zhang B, Pan X, Cobb GP and Anderson TA: microRNAs as oncogenes and tumor suppressors. Dev Biol 302: 1-12, 2007.

6. Wang D, Qiu C, Zhang H, Wang J, Cui Q and Yin Y: Human microRNA oncogenes and tumor suppressors show significantly different biological patterns: from functions to targets. PLoS One 5, 2010.

7. Hong L, Yang Z, Ma J and Fan D: Function of miRNA in controlling drug resistance of human cancers. Curr Drug Targets 14: 1118-1127, 2013.

8. Sun YM, Lin KY and Chen YQ: Diverse functions of miR-125 family in different cell contexts. J Hematol Oncol 6: 6, 2013.

9. Guan Y, Yao H, Zheng Z, Qiu G and Sun K: MiR-125b targets BCL3 and suppresses ovarian cancer proliferation. Int J Cancer 128: 2274-2283, 2011.

10. Huang L, Luo J, Cai Q, et al: MicroRNA-125b suppresses the development of bladder cancer by targeting E2F3. Int J Cancer 128: 1758-1769, 2011.

11. Scott GK, Goga A, Bhaumik D, Berger CE, Sullivan CS and Benz CC: Coordinate suppression of ERBB2 and ERBB3 by enforced expression of micro-RNA miR-125a or miR-125b. J Biol Chem 282: 1479-1486, 2007.

12. Liang L, Wong CM, Ying Q, et al: MicroRNA-125b suppressesed human liver cancer cell proliferation and metastasis by directly targeting oncogene LIN28B2. Hepatology 52: 1731-1740, 2010.

13. Kappelmann M, Kuphal S, Meister G, Vardimon L and Bosserhoff AK: MicroRNA miR-125b controls melanoma progression by direct regulation of c-Jun protein expression. Oncogene 32: 2984-2991, 2013.

14. Liu LH, Li H, Li JP, et al: miR-125b suppresses the proliferation and migration of osteosarcoma cells through down-regulation of STAT3. Biochem Biophys Res Commun 416: 31-38, 2011.

15. Bloomston M, Frankel WL, Petrocca F, et al: MicroRNA expression patterns to differentiate pancreatic adenocarcinoma from normal pancreas and chronic pancreatitis. JAMA 297: 1901-1908, 2007

16. Shi XB, Xue L, Yang J, et al: An androgen-regulated miRNA suppresses Bak1 expression and induces androgen-independent growth of prostate cancer cells. Proc Natl Acad Sci USA 104: 19983-19988, 2007.

17. Vander Heiden M, Cantley LC and Thompson CB: Understanding the Warburg effect: the metabolic requirements of cell proliferation. Science 324: 1029-1033, 2009.

18. Najafov A and Alessi DR: Uncoupling the Warburg effect from cancer. Proc Natl Acad Sci USA 107: 19135-19136, 2010.

19. Zhao Y, Butler EB and Tan M: Targeting cellular metabolism to improve cancer therapeutics. Cell Death Dis 4: e532, 2013.

20. Zhou M, Zhao Y, Ding Y, et al: Warburg effect in chemosensitivity: targeting lactate dehydrogenase-A re-sensitizes taxol-resistant cancer cells to taxol. Mol Cancer 9: 33, 2010.

21. Nakano A, Tsuji D, Miki H, et al: Glycolysis inhibition inactivates $\mathrm{ABC}$ transporters to restore drug sensitivity in malignant cells. PLoS One 6: e27222, 2011.

22. Tong J, Xie G, He J, Li J, Pan F and Liang H: Synergistic antitumor effect of dichloroacetate in combination with 5-fluorouracil in colorectal cancer. J Biomed Biotechnol 2011: 740564, 2011.

23. Singh S, Singh PP, Roberts LR and Sanchez W: Chemopreventive strategies in hepatocellular carcinoma. Nat Rev Gastroenterol Hepatol 11: 45-54, 2014.

24. Abdel-Hamid NM and Morsy MA: Novel biochemical pathways for 5-Fluorouracil in managing experimental hepatocellular carcinoma in rats. J Membr Biol 234: 29-34, 2010. 
25. Yoshida H, Onda M, Tajiri T, et al: Hepatocellular carcinoma responding to chemotherapy with 5-FU. Hepatogastroenterology 47: 1120-1121, 2000.

26. Lupini L, Bassi C, Ferracin M, et al: miR-221 affects multiple cancer pathways by modulating the level of hundreds messenger RNAs. Front Genet 4: 64, 2013.

27. Visone R, Russo L, Pallante P, et al: MicroRNAs (miR)-221 and miR-222, both overexpressed in human thyroid papillary carcinomas, regulate p27Kip1 protein levels and cell cycle. Endocr Relat Cancer 14: 791-798, 2007.

28. Ivanovska I, Ball AS, Diaz RL, et al: MicroRNAs in the miR-106b family regulate $\mathrm{p} 21 / \mathrm{CDKN} 1 \mathrm{~A}$ and promote cell cycle progression. Mol Cell Biol 28: 2167-2174, 2008.

29. Zhou R, Li X, Hu G, Gong AY, Drescher KM and Chen XM: miR-16 targets transcriptional corepressor SMRT and modulates NF-kappaB-regulated transactivation of interleukin-8 gene. PLoS One 7: e30772, 2012.

30. Henson BJ, Bhattacharjee S, O'Dee DM, Feingold E and Gollin SM: Decreased expression of miR-125b and miR-100 in oral cancer cells contributes to malignancy. Genes Chromosomes Cancer 48: 569-582, 2009.
31. Balkhi MY, Iwenofu OH, Bakkar N, et al: miR-29 acts as a decoy in sarcomas to protect the tumor suppressor A20 mRNA from degradation by HuR. Sci Signal 6: ra63, 2013.

32. Li Y, VandenBoom TG II, Wang Z, et al: miR-146a suppresses invasion of pancreatic cancer cells. Cancer Res 70: 1486-1495, 2010

33. Le MT, Teh C, Shyh-Chang N, et al: MicroRNA-125b is a novel negative regulator of p53. Genes Dev 23: 862-876, 2009.

34. Rajabi H, Jin C, Ahmad R, McClary C, Joshi MD and Kufe D: Mucin 1 Oncoprotein Expression Is Suppressed by the miR-125b Oncomir. Genes Cancer 1: 62-68, 2010.

35. Peschiaroli A, Giacobbe A, Formosa A, et al: miR-143 regulates hexokinase 2 expression in cancer cells. Oncogene 32: 797-802, 2013.

36. Kefas B, Comeau L, Erdle N, Montgomery E, Amos S and Purow B: Pyruvate kinase M2 is a target of the tumor-suppressive microRNA-326 and regulates the survival of glioma cells. Neuro Oncol 12: 1102-1112, 2010. 